\title{
Novel Use of Capture-Recapture Methods to Estimate Completeness of Contact Tracing during an Ebola Outbreak, Democratic Republic of the Congo, 2018-2020
}

Jonathan A. Polonsky, Dankmar Böhning, Mory Keita, Steve Ahuka-Mundeke, Justus Nsio-Mbeta, Aaron Aruna Abedi, Mathias Mossoko, Janne Estill, Olivia Keiser, Laurent Kaiser, Zabulon Yoti, Patarawan Sangnawakij, Rattana Lerdsuwansri, Victor J. Del Rio Vilas

Despite its critical role in containing outbreaks, the efficacy of contact tracing, measured as the sensitivity of case detection, remains an elusive metric. We estimated the sensitivity of contact tracing by applying unilist capture-recapture methods on data from the 2018-2020 outbreak of Ebola virus disease in the Democratic Republic of the Congo. To compute sensitivity, we applied different distributional assumptions to the zero-truncated count data to estimate the number of unobserved case-patients with any contacts and infected contacts. Geometric distributions were the best-fitting models. Our results indicate that contact tracing efforts identified almost all ( $n=792,99 \%)$ of case-patients with any contacts but only half ( $n=207,48 \%$ ) of case-patients with infected contacts, suggesting that contact tracing efforts performed well at identifying contacts during the listing stage but performed poorly during the contact follow-up stage. We discuss extensions to our work and potential applications for the ongoing coronavirus pandemic.

Q ontact tracing is the process by which persons who are believed to have come into contact with a confirmed case-patient with an infectious disease during

Author affiliations: World Health Organization, Geneva, Switzerland (J.A. Polonsky); Institute of Global Health, University of Geneva, Geneva (J.A. Polonsky, M. Keita, J. Estill, O. Keiser, L. Kaiser); University of Southampton, Southampton, UK (D. Böhning); World Health Organization Regional Office for Africa, Brazzaville, Republic of the Congo (M. Keita, Z. Yoti); Institut National de Recherche Biomédicale, Kinshasa, Democratic Republic of the Congo (S. Ahuka-Mundeke, M. Mossoko); Ministere de la Santé Publique, Kinshasa (J. Nsio-Mbeta, A. Aruna Abedi); Thammasat University, Bangkok, Thailand (P. Sangnawakij, R. Lerdsuwansri); World Health Organization South East Asia Regional Office, New Delhi, India (V.J. Del Rio Vilas)

DOI: https://doi.org/10.3201/eid2712.204958 the infectious period are located and checked for the presence of the infection or disease. Under traditional approaches, contact tracing involves 3 distinct steps: contact identification, in which potential contacts are identified through interview with the primary case-patient; contact listing, in which those identified contacts are listed and communication established with them; and contact follow-up, in which those listed contacts are monitored for presence of infection or onset of disease over a predefined period (1).

Because of its important role in case detection to monitor and curtail chains of transmission, contact tracing often forms part of the public health response to directly transmitted infectious diseases (2). Recently, contact tracing has received widespread attention because of its critical role in the response to outbreaks of diphtheria (3), Ebola virus disease (EVD) (4-6), and the ongoing coronavirus disease (COVID-19) pandemic $(7,8)$.

During 2018-2020, the Democratic Republic of the Congo (DRC) experienced its 10th and largest EVD outbreak, the second largest ever experienced globally (9). EVD is a disease caused by viruses of the genus Ebolavirus, family Filoviridae. Zoonotic spillover events from the animal reservoir have led to large, explosive outbreaks in West and Central Africa in recent years (9-12). Owing to the high pathogenicity and virulence of Ebola virus, an elimination control strategy is always adopted, aiming to ensure that all case-patients are identified, isolated, and treated promptly after disease onset, thereby limiting the opportunity for onward community spread. Although contact tracing is a central pillar of control (13), no standardized methods have been established to assess a critical aspect of performance, its sensitivity 
(i.e., the ability to detect all contacts and secondary infections resulting from case-patients).

One approach to quantifying this metric is to employ capture-recapture (CRC) methods $(14,15)$. Broadly, this family of methodologic approaches enables researchers to quantify any unit of interest missing from lists and subsequently estimate the sensitivity of the surveillance effort and the probability of detection. Although CRC has previously been used to estimate the number of unobserved cases of disease $(16,17)$, such approaches typically rely on comparison of multiple lists, which are generally not available for contact lists. Therefore, we describe the application of a unilist capture-recapture approach (15) to quantifying the number of unobserved case-patients and contacts and describe their sociodemographic profile, helping to identify plausible risk factors that can be used to target limited resources at those unobserved case-patients most likely to generate onward transmission. More precisely, we aimed to address 2 questions, from which we can derive contact tracing sensitivity estimates: how many case-patients with any contacts did contact tracing miss, and how many case-patients with infected contacts did contact tracing miss?

\section{Materials and Methods}

\section{Study Participants}

We included all confirmed and probable EVD case-patients and contacts (classified according to standardized case definitions $[18,19]$ ) identified in Beni Health Zone, DRC, during July 31, 2018-April 26, 2020. Casepatients were principally detected through 3 identification mechanisms: passive detection at healthcare facilities from persons manifesting symptoms consistent with EVD, house-to-house active case-finding by community health workers, and tracing the contacts of EVD case-patients. Contact tracing was coordinated by the DRC Ministry of Public Health, with support from the World Health Organization, and conducted by locally recruited teams of contact-tracers. Upon detection of a case, efforts to identify and list the case-patient's contacts were undertaken.

For case-patients, our data contain basic information on sociodemographic characteristics (e.g., age, sex, and DRC Health Area of residence) and dates of disease onset and isolation. For contacts, our data contain similar sociodemographic information and information on the daily follow-up and final status of the contact (either "completed the 21 days follow-up," "confirmed as EVD case-patient," "lost to follow-up," "never seen," or "died during follow-up"). Contacts recorded as "confirmed as EVD case-patients" were those identified by the contact tracing teams during the course of their work. EVD was assumed to be the cause of death for contacts recorded as "died during follow-up" because of the short interval between their contact with an EVD case-patient and their death.

\section{Exploratory Data Analysis}

We determined the distribution of case-patients according to age, sex, and timing of disease onset. We used the Wilcoxon test to explore differences in continuous variables and the $\chi^{2}$ test for categoric variables to determine the distribution of the number of contacts per case-patient between 2 distinct epidemic waves. Overdispersion (i.e., superspreading) in the offspring distribution of secondary case-patients arising from infectious persons may have profound effects on control strategies in low-resource settings $(20,21)$, and we describe the extent of this phenomenon in 2 ways: first, by assessing the proportion of infectious persons linked to $80 \%$ of onward transmission using methods described by Endo et al. (22); and second, by estimating the dispersion parameter $(k)$ using methods described by Althaus (23).

We used a multivariable logistic regression model to explore risk factors associated with loss to follow-up, in which previously successfully traced contacts (i.e., those identified, listed, and among whom follow-up has begun) become untraceable at some point during the 21-day follow-up period. In such instances, contacts unable to be traced for 3 consecutive days are recorded as having been lost to followup, and no further attempts at tracing are made. To explore characteristics of case-patients with infected contacts, we calculated the mean number of contacts, mean age, and sex ratio of case-patients with $\geq 1$ listed contact (among whom we can be confident that at least a minimal investigation was conducted), according to 3 categories: those with no infected contacts identified, those with exactly 1 contact, and those with $\geq 2$ contacts.

\section{CRC Modeling}

We classified the observed case-patients according to their number of listed contacts (either exactly 0 or $\geq 1$ contact), further classifying this latter category according to the number of infected contacts observed (either exactly 0 or $\geq 1$ contact). For each detected case, the contact tracing process generates a list of persons fitting the definition for a contact (Appendix, https:/ / wwwnc.cdc.gov/EID/article/27/12/204958-App1.pdf), some of whom may themselves have been infected and will eventually become secondary 
case-patients. From this list, frequency distributions of case-patients with any listed contacts, and of casepatients with infected contacts, can be generated by first excluding (truncating) those case-patients with 0 contacts. For example, the data can be binned into the number of case-patients with exactly 1 contact $\left(f_{1}\right), 2$ contacts $\left(f_{2}\right)$, and so on, to the number of case-patients with the maximum number of contacts $\left(f_{m}\right)$. Statistically, this process leads to a 0-truncated observed count distribution of case-patients with $\geq 1$ contact. By applying a unilist CRC approach designed to estimate unobserved population sizes using the distribution of count data within single lists (15), we can infer $f_{0^{\prime}}$ the number of unobserved case-patients with $\geq 1$ contact. Associated with the observed frequencies $\left(f_{1}\right.$, $\left.f_{2}, \ldots, f_{m}\right)$ and unobserved $f_{0}$ are probabilities $p_{1}, p_{2}, \ldots$, $p_{m}$ and $p_{0}$ that inform the probability of identifying a case-patient with exactly $1,2, \ldots, m$ and 0 contacts, respectively. A conventional approach assumes that the frequencies arise from a discrete distribution such as the Poisson, where

$$
p_{0}=e^{-\lambda}, p_{1}=\frac{e^{-\lambda} \lambda}{1 !}, p_{2}=\frac{e^{-\lambda} \lambda^{2}}{2 !}, \ldots, p_{m}=\frac{e^{-\lambda} \lambda^{m}}{m !} \text {. }
$$

Other common distributions are the negative binomial and the geometric distribution. The geometric distribution has probabilities $p_{0}=p, p_{1}=p(1-p), p(1-$ $p)^{2} \ldots p_{m}=p(1-p)^{m}$, where $p$ is a probability parameter. Poisson and geometric are special cases of the negative binomial distribution, which provides a flexible model family (Appendix). Because the observed distribution contains only positive numbers of contacts, we need to consider the associated zero-truncated distribution $p_{1} /\left(1-p_{0}\right), p_{2} /\left(1-p_{0}\right), \ldots p_{m} /\left(1-p_{0}\right)$. In other words, we assume that the number of observed contacts among case-patients who actually had contacts follows a parametric distribution (although nonparametric approaches are possible $[15,24,25])$, find the best-fitting zero-truncated distribution of case-patients with $\geq 1$ observed contact (we explore the zero-truncated Poisson, negative binomial, and geometric distributions [Appendix]), and use the estimated probability $p_{0}$ of not observing a case-patient with contacts (calculated from the best-fitting distribution) to inform standard population estimators. We use the Horvitz-Thompson estimator to estimate $f_{0^{\prime}}$ the unobserved number of case-patients:

$$
\widehat{f_{0}}=n \frac{p_{0}}{1-p_{0}}
$$

where $n$ is the number of observed case-patients with $\geq 1$ observed contact and $p_{0}$ is as previously defined. The Horvitz-Thompson estimator provides an unbiased estimate of $f_{0}$, provided that $p_{0}$ is correctly specified; hence, using a correctly-specified distribution for the number of observed contacts is important. We use maximum likelihood for model fitting, selecting the model with the smallest Akaike information criteria (AIC) and Bayesian information criteria (BIC) (Appendix).

To estimate $95 \%$ CIs, we use a parametric bootstrap, described as follows. Suppose that $N$ is the estimated size of the (observed and unobserved) population under a fitted model. We generate $B$ samples of size $N_{b}$ using the fitted model and its estimated parameter or parameters. For each sample, all zeros are truncated and the size estimate $N_{b}$ computed, for each of the samples $b=1, \ldots, B$. We chose $B=10,000$ to minimize bootstrap simulation random error. We constructed $95 \%$ CIs by using the 2.5th percentile of the distribution of $N_{b}$ as the lower end and the 97.5 th percentile as the upper end.

\section{Results}

\section{Exploratory Data Analysis}

We identified 913 confirmed and 10 probable EVD case-patients in Beni Health Zone. The contact tracing process listed 80,556 contacts, of whom 6,375 were duplicates, having been listed as the contact of $>1$ case-patient, resulting in 74,181 contacts to trace. In discussion with contact tracing teams, duplicates were identified by matching name and residential location; for operational reasons, these persons were recorded as a contact of only the earliest-identified primary case-patient with whom they were associated. More than half of case-patients for whom sex and age information were available were women and girls ( $\mathrm{n}=515$ [55.8\%]); median age for all casepatients was 25 years (interquartile range [IQR] 13-38 years). Most contacts $(64,545$ [87.0\%]) were successfully traced, leading to the detection of 396 secondary case-patients. The median delay between last contact with the primary case-patient and first contact by the contact tracing teams was 4 days (IQR 3-6 days).

Disease onset dates spanned the period from July 31, 2018, to April 26, 2020, and was bimodally distributed, showing 2 waves that peaked in October 2018 and June 2019 (Figure 1, panel A). The second wave followed a period of insecurity in this conflict-affected area that severely hampered response activities (26).

The median number of contacts among all casepatients was 61 (IQR 18-120), but this number was significantly lower during the first wave than the second (34 vs. 80; $p<0.001)$. Case-patients infected in the first wave were more likely to have 0 listed contacts than those in the second wave ( $31.3 \%$ vs. $9.6 \%$; $<<0.001$ 
by $\chi^{2}$ test), and second-wave case-patients were more likely to have a large number $(>100)$ of contacts (Figure 1, panel B). A total of 792 case-patients (85.8\%) reported $\geq 1$ contact (Figure 2, 3), among whom the median number of contacts was 74 (IQR 36-134) and the mean number of contacts was 102.

A total of 64,545 contacts $(87.0 \%)$ were successfully traced, of whom 308 were confirmed as EVD casepatients and 88 died during follow-up. Therefore, the inferred total number of infected contacts was 396 $(308+88)$, or $0.7 \%$ of the contacts successfully traced to completion of the follow-up period. Precise detail on the mechanism of identification of confirmed casepatients among contacts is not available; although we assume these infected contacts were identified by contact tracers during follow-up, the role of other surveillance activities cannot be excluded.

We observed substantial overdispersion in the offspring distribution of secondary case-patients; $80 \%$ of onward transmission was linked to only $13.9 \%$ (95\% CI $11.4 \%-16.2 \%)$ of primary case-patients, and all secondary case-patients concentrated among the contacts of $207(22.4 \%)$ primary case-patients. Fur- ther, only $99(10.7 \%)$ primary case-patients led to $>1$ secondary case-patient (Figure 2, 4). We estimated $k$ as 0.27 (95\% CI 0.20-0.33).

Male contacts had slightly (but statistically significantly) greater odds of being lost to follow-up (odds ratio [OR] 1.06, 95\% CI 1.01-1.11) (Table 1). Contacts in older age groups had significantly greater odds of being lost to follow-up compared with contacts in the youngest age group (0-15 years). We observed the greatest effect among contacts $\geq 60$ years of age (OR 1.65, 95\% CI 1.47-1.86) and a marginally smaller effect among contacts 45-59 years of age (OR 1.55, 95\% CI 1.43-1.69). Conversely, contacts traced during the second wave had lower odds of being lost to follow-up (OR 0.83, 95\% CI 0.79-0.88).

\section{CRC Modeling}

\section{Completeness of Contract Tracing for Case-Patients} with $>1$ Listed Contact

Among case-patients with $\geq 1$ contact listed, the bestfitting distribution of the count of case-patients with any contacts was given by the zero-truncated geomet-
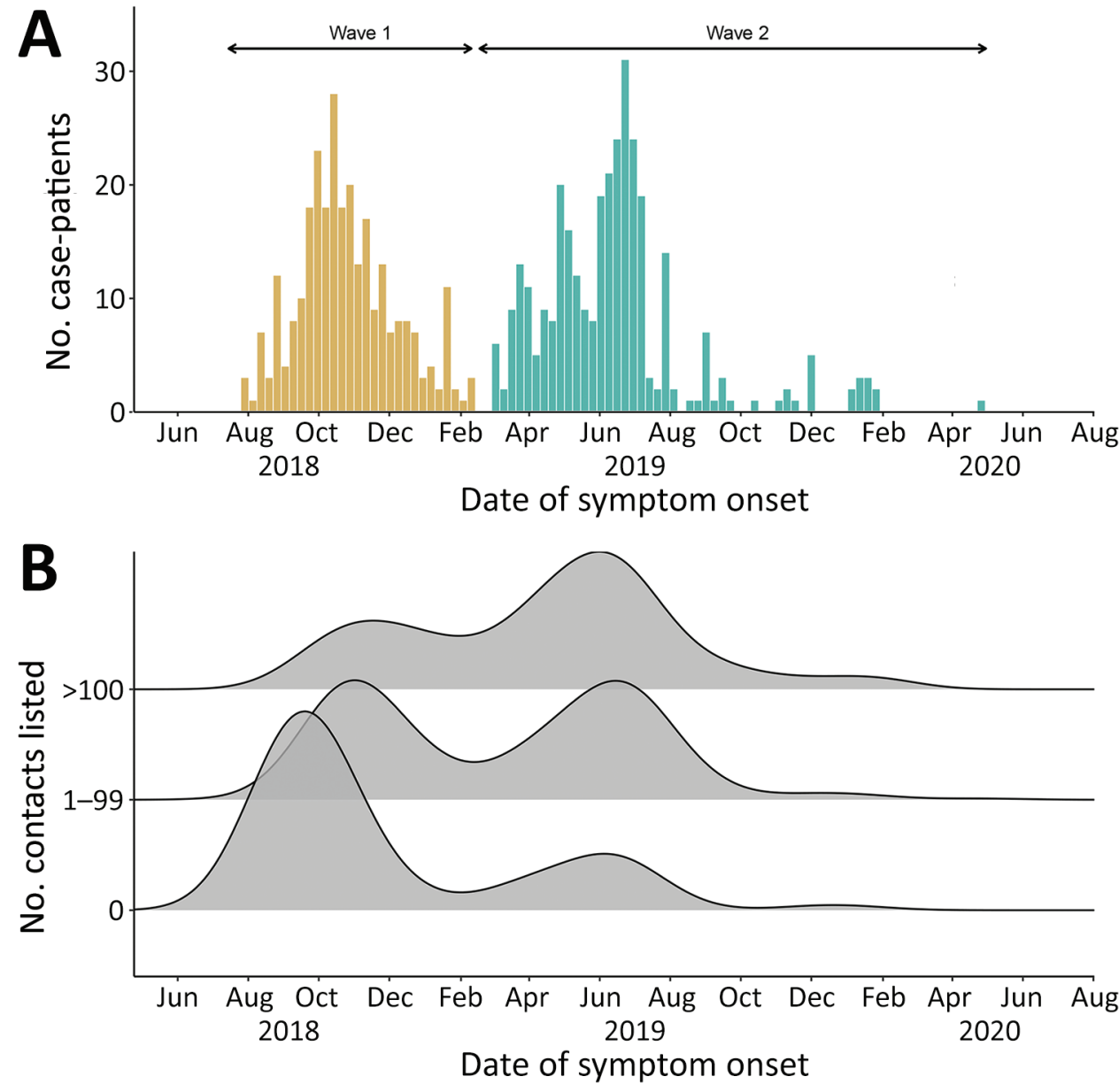

Figure 1. Epidemic curve and symptom onset dates among Ebola virus disease casepatients, Beni Health Zone, Democratic Republic of the Congo, July 31, 2018-April 26, 2020. A) Epidemic curve by date of symptom onset. Case-patients and contacts were divided into 2 epidemic waves, according to the date of symptom onset among case-patients (first wave, July 31, 2018-February 28, 2019; second wave, March 1, 2019-April 26, 2020). B) Distribution of dates of symptom onset among case-patients, by number of listed contacts. Data were smoothed by using a nonparametric (Gaussian) kernel-based estimate, with automatic bandwidth selection (37.6 days). 
ric model, which produced the lowest AIC and BIC (Appendix Table 1). This distribution was very longtailed (Figure 5), indicating that most case-patients with contacts were successfully detected, given that with an increasing mean of any count distribution, the probability for a zero count becomes smaller. This pattern is observed from the expression of the geometric distribution, described previously, where for $x=0$ (i.e., the zero count), its estimated probability $p_{0}$ resolves the equation

$$
\frac{1}{1+\mu}\left(\frac{\mu}{1+\mu}\right)^{x}
$$

to return

$$
\frac{1}{1+\mu}
$$

where $\mu$ is the mean of the geometric model; the larger the mean, the smaller the probability of $x=0$.

We estimated $f_{0}$ (the unobserved number of casepatients with any contacts $)=8(95 \% \mathrm{CI}=8-10)$, where sample size $(n)$ was 792 and $p_{0}$ was found as 0.01 . The sensitivity of contact tracing to detect case-patients with any contacts was therefore $792 /(792+8)=0.99 \%$ (95\% CI $0.99 \%-0.99 \%)$. We observed no difference in sensitivity by epidemic wave (wave $1=0.99 \%$ [95\% CI $0.99 \%-0.99 \%$ ]; wave $2=0.99$ [95\% CI 0.99-0.99]).

\section{Completeness of Contact Tracing for Case-Patients with Infected Contacts}

Among case-patients with infected contacts, the best-fitting distribution of the count of case-patients with infected contacts was again given by the zero-truncated geometric model, which produced the lowest AIC and BIC (Appendix Table 1). This

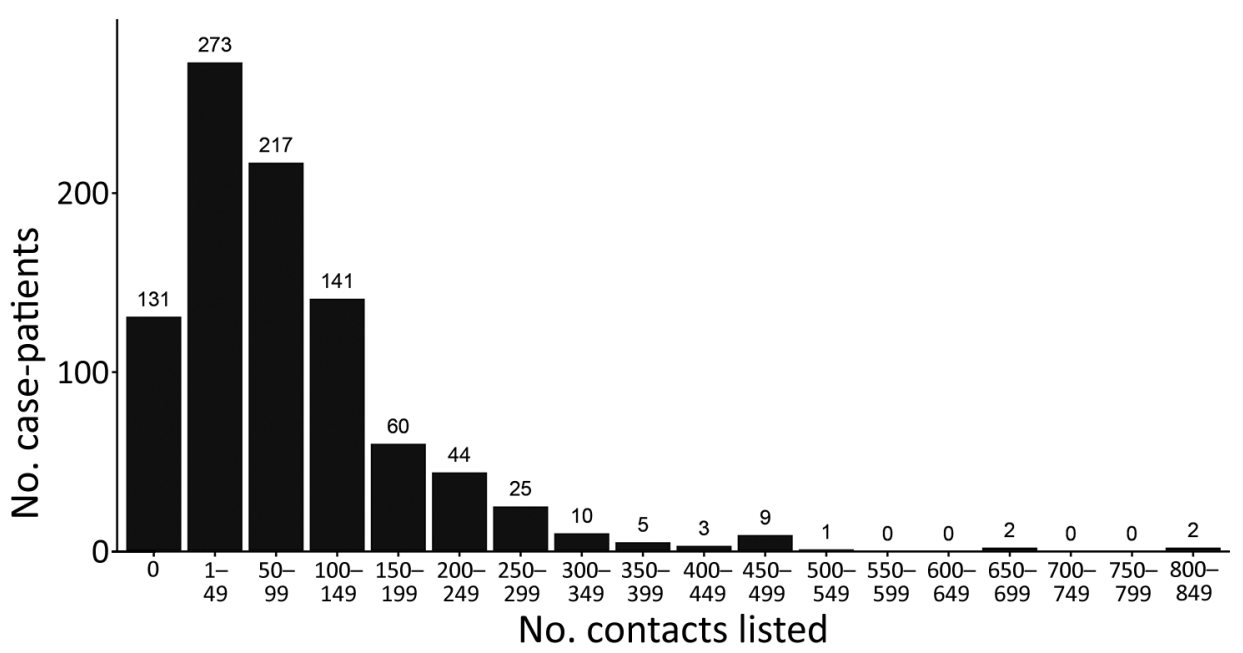

923 total casepatients observed

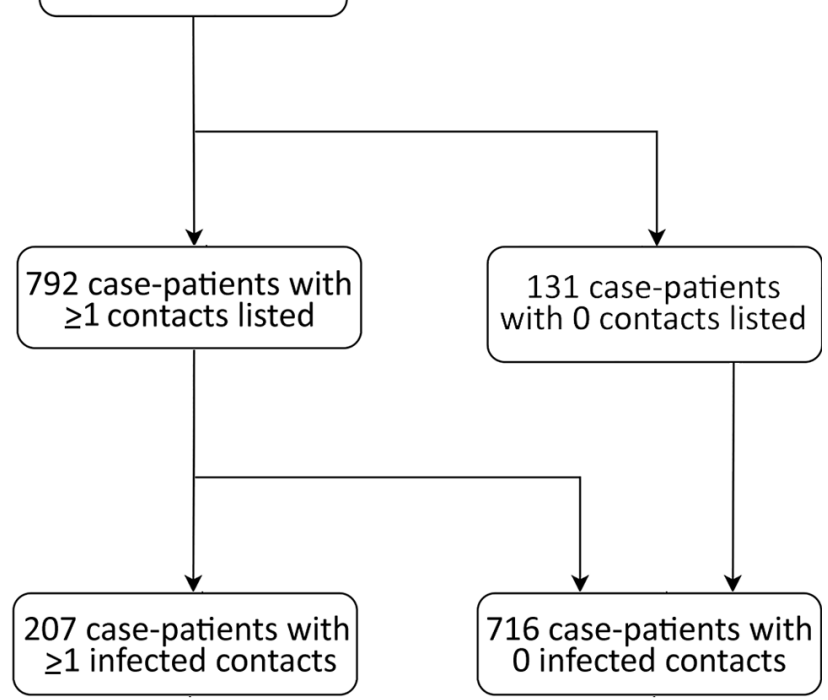

Figure 2. Flowchart showing breakdown of observed casepatients by number of listed and infected contacts among Ebola virus disease case-patients, Beni Health Zone, Democratic Republic of the Congo, July 31, 2018-April 26, 2020.

distribution is concentrated on the lower counts from 1 to 4 (Figure 6), indicating that a substantial proportion of case-patients with infected contacts may not have been detected.

We estimated $f_{0}$ (the unobserved number of casepatients with infected contacts) $=227$ (95\% CI 171241), where sample size $(n)$ was 207 and $p_{0}$ was found as 0.52 . The sensitivity of contact tracing to detect case-patients with infected contacts was therefore $207 /(207+227)=0.49 \%(95 \%$ CI $0.43 \%-0.55 \%)$. We observed a statistically significant difference in sensitivity by epidemic wave, with lower sensitivity

Figure 3. Frequency distribution of Ebola virus disease case-patients, by number of listed contacts, Beni Health Zone, Democratic Republic of the Congo, July 31, 2018-April 26, 2020. 


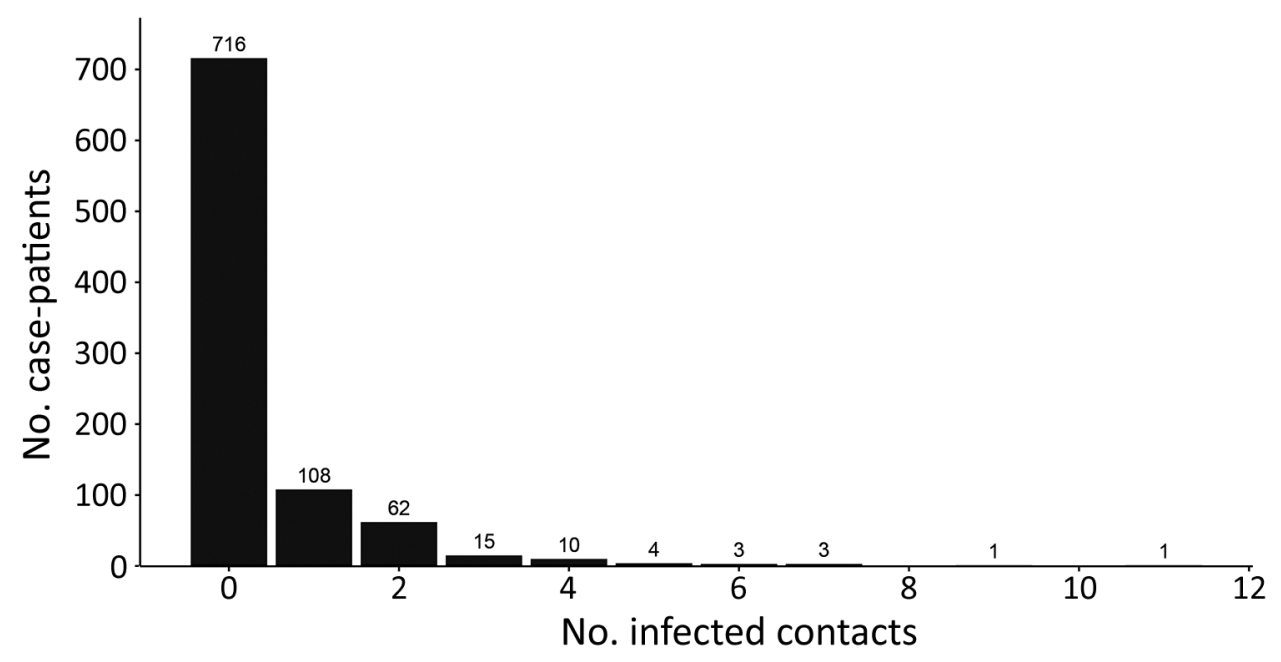

Figure 4. Frequency distribution of Ebola virus disease case-patients, by number of infected contacts, Beni Health Zone, Democratic Republic of the Congo, July 31, 2018-April 26, 2020. during wave $1(0.24 \%$ [95\% CI $=0.11 \%-0.38 \%])$ than during wave $2(0.48 \%$ [95\% CI $=0.40 \%-0.56 \%])$. Among the 792 case-patients with $\geq 1$ listed contact, those with 0 infected contacts had fewer contacts overall, were slightly older, and were slightly more likely to be women or girls compared with the other groups (Table 2).

\section{Discussion}

Our findings suggest that contract tracing efforts were very successful at identifying case-patients with $\geq 1$ contact but much less successful at identifying case-patients with contacts who later had EVD symptoms. This finding is unsurprising, given that the investigation component (typically by interview with case-patients under treatment, their caregivers, or both) is easier to conduct than the tracing component (typically requiring daily visits to a large number of difficult-to-locate and mobile persons). This difference has important implications, because infected contacts contribute to ongoing chains of transmission when case investigation and contract tracing is inadequate; to prioritize scarce resources, control efforts should target those casepatients among whose contacts secondary infections arise $(20,21,27)$. A high proportion of case-patients listed $\geq 1$ contact $(\approx 85 \%)$, compared with $27 \%$ during an EVD outbreak in Liberia (28) and 44\% during an EVD outbreak in Sierra Leone (27), suggesting that lessons about enhancing the quality of contract tracing were learned from previous EVD outbreaks $(4,5,27,28)$.

Case-patients with infected contacts had more contacts on average, which may result from 3 possible explanations. First, case-patients with more contacts are more likely to have $\geq 1$ infected contact among these. Second, fewer overall listed contacts may be the result of poorly conducted case investigations. We found some evidence in support of this; the mean number of contacts increased as the

Table 1. Multivariable logistic regression for predictors of loss to follow-up of contacts of Ebola virus disease case-patients, Beni Health Zone, Democratic Republic of the Congo, July 31, 2018-April 26, 2020*

\begin{tabular}{|c|c|c|c|c|c|}
\hline \multirow[b]{2}{*}{ Independent variable } & \multirow[b]{2}{*}{ No. contacts } & \multicolumn{2}{|c|}{ Unadjusted } & \multicolumn{2}{|c|}{ Adjusted } \\
\hline & & OR $(95 \% \mathrm{Cl})$ & $p$ value & OR $(95 \% \mathrm{Cl})$ & $p$ value \\
\hline \multicolumn{6}{|l|}{ Sex } \\
\hline $\mathrm{F}$ & 41,349 & Referent & & Referent & \\
\hline $\mathrm{M}$ & 37,296 & $1.07(1.02-1.12)$ & 0.003 & $1.06(1.01-1.11)$ & 0.013 \\
\hline \multicolumn{6}{|l|}{ Age group, $y$} \\
\hline $0-14$ & 20,616 & Referent & & Referent & \\
\hline $15-29$ & 26,142 & $1.18(1.11-1.25)$ & $<0.001$ & $1.19(1.12-1.27)$ & $<0.001$ \\
\hline $30-44$ & 17,665 & $1.16(1.09-1.24)$ & $<0.001$ & $1.18(1.10-1.26)$ & $<0.001$ \\
\hline $45-59$ & 6,157 & $1.56(1.43-1.70)$ & $<0.001$ & $1.55(1.43-1.69)$ & $<0.001$ \\
\hline$>60$ & 2,599 & $1.64(1.46-1.84)$ & $<0.001$ & $1.65(1.47-1.86)$ & $<0.001$ \\
\hline \multicolumn{6}{|l|}{ Epidemic wave† } \\
\hline First wave & 14,374 & Referent & & Referent & \\
\hline Second wave & 66,182 & $0.85(0.81-0.90)$ & $<0.001$ & $0.83(0.79-0.88)$ & $<0.001$ \\
\hline
\end{tabular}

*OR, odds ratio

†Contacts were divided into 2 epidemic waves according to the date of symptom onset of their associated primary case-patient (first wave, July 31,2018

February 28, 2019; second wave, March 1, 2019-April 26, 2020). 
Table 2. Distribution of Ebola virus disease case-patients, their median age and sex ratio, and mean total number of contacts, grouped by number of infected contacts, among case-patients with $\geq 1$ listed contact, Beni Health Zone, Democratic Republic of the Congo, July 31, 2018-April 26, 2020

\begin{tabular}{lcccc}
\hline No. infected contacts & No. case-patients & $\begin{array}{c}\text { Median age of } \\
\text { case-patients, } y\end{array}$ & $\begin{array}{c}\text { \% Women and girls } \\
\text { among case-patients }\end{array}$ & $\begin{array}{c}\text { Mean (95\% Cl) total } \\
\text { number of contacts }\end{array}$ \\
\hline 0 & 585 & 28.2 & 59.5 & $85.7(79.1-92.4)$ \\
1 & 108 & 23.6 & 56.7 & $122(102.0-141.0)$ \\
2 & 99 & 25.6 & 54.6 & $174(144.0-204.0)$ \\
\hline
\end{tabular}

epidemic progressed, indicating possible improvements in case investigation quality over time as staff became more accustomed to the procedure and community trust and engagement in the response improved (29). Third, case-patients with infected contacts may differ from other case-patients; in this study, such case-patients were younger and more likely to be men or boys, which are demographic factors previously shown to affect transmission of EVD and other diseases (30-32). Case-patients with more contacts have been shown to play a greater role in disease transmission and are more likely to have infected contacts $(33,34)$. This tendency is particularly true of diseases that demonstrate heterogeneous transmission, including EVD and COVID-19, and our results suggest a high degree of overdispersion and superspreading, consistent with what has previously been reported during large EVD outbreaks (23). Overdispersion can lead to rapid expansion, particularly among hidden chains of transmission, and a promising area of research is to identify correlates of superspreading to better target limited resources for greatest impact. Previous research suggests that if highly infectious persons can be predictively identified and targeted, the efficiency of control can be greatly enhanced, such that focusing half of all control effort on the most infectious $20 \%$ of case-patients can improve effectiveness up to 3 -fold $(20,21)$.

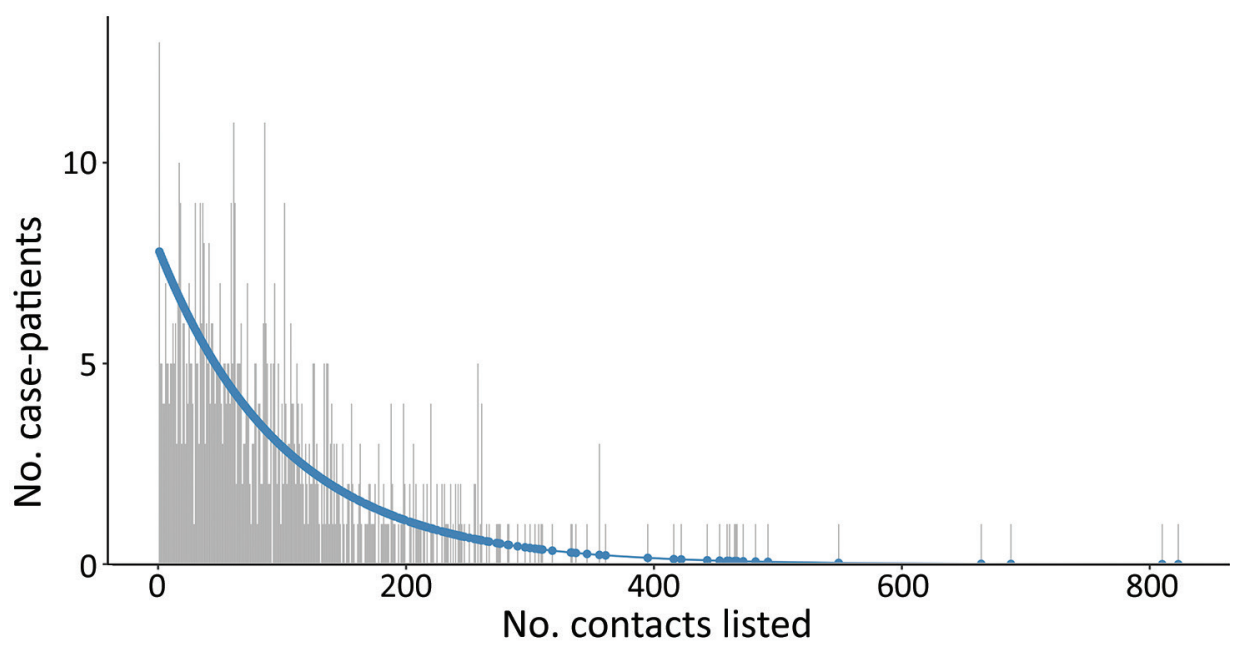

Although estimating the number of unobserved case-patients with (infected) contacts is possible, identifying whether these case-patients have been misclassified as having 0 (infected) contacts or if they were undetected by the surveillance system in general is not possible. However, the greater probability of having 0 contacts listed during the first epidemic wave suggests substantial misclassification and suboptimal performance in the period during which surveillance activities were being established, as reported during previous EVD outbreaks $(4,27,28)$. The sensitivity of contact tracing to detect case-patients with infected contacts was lower, and loss to followup greater, during the first epidemic wave, indicating quality improvements of this activity over time, either because the ability to conduct contact follow-up was hampered by the insecurity experienced during the first wave or because of greater familiarity with, and acceptance of, the process among contact tracing staff and the local population during later efforts.

Although the method we describe proposes a robust framework to assess the sensitivity of contact tracing, limitations include that no standard list of contacts against which to validate this method exists. However, the method itself has been validated to estimate actual population size in various other settings (25). The dataset does not permit the distinction between case-patients who were confirmed to have no contacts after a thorough case investigation and case-
Figure 5. Observed (gray) and fitted (geometric; blue) zerotruncated distribution of the total number of contacts for casepatients with $\geq 1$ contact listed, Beni Health Zone, Democratic Republic of the Congo, July 31 , 2018-April 26, 2020.. 


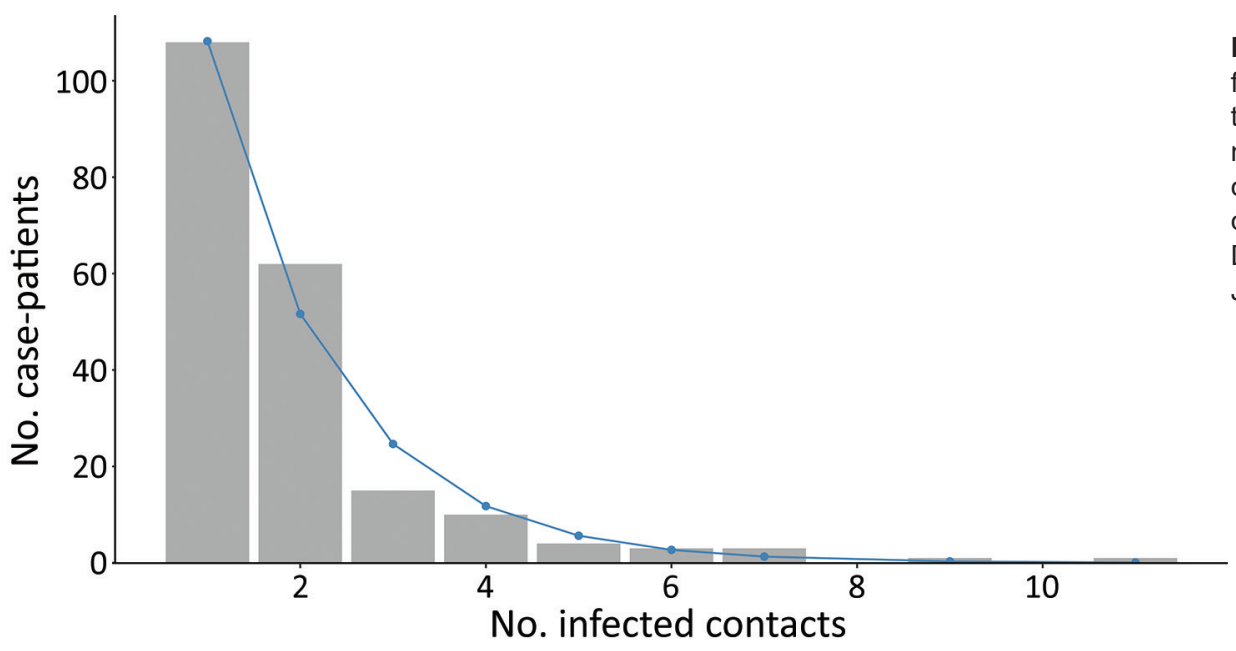

Figure 6. Observed (gray) and fitted (geometric; blue) zerotruncated distribution of the total number of infected contacts for case-patients with $\geq 1$ infected contact listed, Beni Health Zone, Democratic Republic of the Congo, July $31,2018-A p r i l ~ 26,2020$.

patients having no listed contacts because of no (or inadequate) case investigation. However, our method may help to identify the magnitude of the misclassification arising from this limitation. The inferences made are exclusively informed by the definition of case-patients as defined by contact tracing protocols; for example, our results would not inform the sensitivity of contact tracing as applied to asymptomatic EVD case-patients if these persons are not part of the testing strategy.

Differences in performance between contact tracers could result in strong heterogeneity in the count distribution, which might be detectable. For this reason, we applied Chao's estimator (which allows for heterogeneity), and only if this was significantly different from the model-based estimate would we consider that an issue exists. In our results, we did not observe such a difference (Appendix Tables 2, 3). Finally, we have not adjusted for observed heterogeneity, such as age, sex, profession, geographic location of the case-patients, and delays in the contact tracing process. Further work is planned to incorporate such considerations.

In conclusion, contact tracing is crucial to containing certain disease outbreaks. However, as with many surveillance activities, contact tracing has the potential to suffer reduced effectiveness from underreporting and poor sensitivity $(4,27,28)$. The consequences of poor ascertainment and misclassification can be disastrous, potentially creating explosive expansion among hidden chains of transmission, particularly during containment and deescalation phases.

We have described a novel application of CRC models to estimate a crucial yet elusive performance indicator of a key component of the public health response to epidemics, namely the sensitivity of contact tracing, as applied to a recent outbreak of EVD. The method demonstrated that most casepatients with any contacts were observed, suggesting that the case investigation component of contact tracing performed well, whereas less than half of case-patients with infected contacts were observed, suggesting that the contact follow-up component of contact tracing performed poorly in this setting. The approach described can be used to assess the sensitivity of contact tracing for any disease, including COVID-19, for which contact tracing has been identified as a crucial component of response activities.

\section{Acknowledgments}

We thank the people of the DRC's Beni Health Zone for their participation in conducting this study. We acknowledge the enormous dedication of the various organizations and persons that responded and provided healthcare to this population and supported the public health response during this outbreak.

The authors received no specific funding for this work. The authors alone are responsible for the views expressed in this article, and they do not necessarily represent the views, decisions, or policies of the institutions with which they are affiliated.

\section{About the Author}

Mr. Polonsky is an epidemiologist with the World Health Organization, Geneva, Switzerland. His research interests include various aspects of public health response in humanitarian and emergency settings, with a primary focus on infectious disease outbreaks and monitoring the health impact of crises. 


\section{References}

1. World Health Organization. Infection prevention and control: contact tracing [cited 2021 May 10]. https:/ / www. who.int/news-room/q-a-detail/contact-tracing

2. Polonsky JA, Baidjoe A, Kamvar ZN, Cori A, Durski K, Edmunds WJ, et al. Outbreak analytics: a developing data science for informing the response to emerging pathogens. Philos Trans R Soc Lond B Biol Sci. 2019;374:20180276. https://doi.org/10.1098/rstb.2018.0276

3. Polonsky JA, Ivey M, Mazhar MKA, Rahman Z, le Polain de Waroux O, Karo B, et al. Epidemiological, clinical, and public health response characteristics of a large outbreak of diphtheria among the Rohingya population in Cox's Bazar, Bangladesh, 2017 to 2019: a retrospective study. PLoS Med. 2021;18:e1003587. https://doi.org/10.1371/journal.pmed.1003587

4. Swanson KC, Altare C, Wesseh CS, Nyenswah T, Ahmed T, Eyal N, et al. Contact tracing performance during the Ebola epidemic in Liberia, 2014-2015. PLoS Negl Trop Dis. 2018;12: e0006762. https:// doi.org/10.1371/journal.pntd.0006762

5. Polonsky J, Mboussou F, Haskew C, le Polain de Waroux O, Belizaire MRD, Mondonge V, et al. Lessons learnt from Ebola virus disease surveillance in Équateur Province, May-July 2018. Wkly Epidemiol Rec. 2019;5 [cited 2020 Dec 28]. https://apps.who.int/iris/handle/10665/279754

6. Sikakulya FK, Mulisya O, Munyambalu DK, Bunduki GK. Ebola in the eastern Democratic Republic of Congo: One Health approach to infectious disease control. One Health. 2019;9:100117. https:// doi.org/10.1016/j.onehlt.2019.100117

7. Keeling MJ, Hollingsworth TD, Read JM. Efficacy of contact tracing for the containment of the 2019 novel coronavirus (COVID-19). J Epidemiol Community Health. 2020;74:861-6.

8. Althoff KN, Coburn SB, Nash D. Contact tracing: essential to the public health response and our understanding of the epidemiology of coronavirus disease 2019. Clin Infect Dis. 2020;71:1960-1. https://doi.org/10.1093/cid/ciaa757

9. Ilunga Kalenga O, Moeti M, Sparrow A, Nguyen V-K, Lucey D, Ghebreyesus TA. The ongoing Ebola epidemic in the Democratic Republic of Congo, 2018-2019. N Engl J Med. 2019;381:373-83. https://doi.org/10.1056/NEJMsr1904253

10. Aylward B, Barboza P, Bawo L, Bertherat E, Bilivogui $P$, Blake I, et al.; WHO Ebola Response Team. Ebola virus disease in West Africa - the first 9 months of the epidemic and forward projections. N Engl J Med. 2014;371:1481-95. https:/ / doi.org/10.1056/NEJMoa1411100

11. Polonsky JA, Wamala JF, de Clerck H, Van Herp M, Sprecher A, Porten K, et al. Emerging filoviral disease in Uganda: proposed explanations and research directions. Am J Trop Med Hyg. 2014;90:790-3. https:/ / doi.org/ 10.4269/ajtmh.13-0374

12. Barry A, Ahuka-Mundeke S, Ahmed YA, Allarangar $Y$, Anoko J, Archer BN, et al.; Ebola Outbreak Epidemiology Team. Outbreak of Ebola virus disease in the Democratic Republic of the Congo, April-May, 2018: an epidemiological study. Lancet. 2018;392:213-21. https://doi.org/10.1016/ S0140-6736(18)31387-4

13. World Health Organization. Implementation and management of contact tracing for Ebola virus disease [cited 2021 Oct 6]. https://www.who.int/publications/i/item/ WHO-EVD-Guidance-Contact-15.1

14. Vergne T, Del Rio Vilas VJ, Cameron A, Dufour B, Grosbois V. Capture-recapture approaches and the surveillance of livestock diseases: a review. Prev Vet Med. 2015;120:253-64. https://doi.org/10.1016/j.prevetmed.2015.04.003

15. Bohning D, Heijden PGM, Bunge J, editors. Capturerecapture methods for the social and medical sciences. London: Chapman and Hall/CRC; 2017.
16. Gignoux E, Idowu R, Bawo L, Hurum L, Sprecher A, Bastard M, et al. Use of capture-recapture to estimate underreporting of Ebola virus disease, Montserrado County, Liberia. Emerg Infect Dis. 2015;21:2265-7. https:/ / doi.org/ 10.3201/eid2112.150756

17. Isanaka S, Hedt-Gauthier BL, Salou H, Berthé F, Grais RF, Allen BGS. Active and adaptive case finding to estimate therapeutic program coverage for severe acute malnutrition: a capture-recapture study. BMC Health Serv Res. 2019;19:967. https:/ / doi.org/10.1186/s12913-019-4791-9

18. Medley AM, Mavila O, Makumbi I, Nizeyemana F, Umutoni A, Balisanga $\mathrm{H}$, et al. Case definitions used during the first 6 months of the 10th Ebola virus disease outbreak in the Democratic Republic of the Congo-four neighboring countries, August 2018-February 2019. MMWR Morb Mortal Wkly Rep. 2020;69:14-9. https://doi.org/10.15585/ mmwr.mm6901a4

19. World Health Organization. Case definition recommendations for Ebola or Marburg virus diseases. 2014 [cited 2021 Oct 6]. https://www.who.int/csr/resources/publications/ebola/ ebola-case-definition-contact-en.pdf

20. Woolhouse MEJ, Dye C, Etard J-F, Smith T, Charlwood JD, Garnett GP, et al. Heterogeneities in the transmission of infectious agents: implications for the design of control programs. Proc Natl Acad Sci U S A. 1997;94:338-42. https://doi.org/10.1073/pnas.94.1.338

21. Lloyd-Smith JO, Schreiber SJ, Kopp PE, Getz WM. Superspreading and the effect of individual variation on disease emergence. Nature. 2005;438:355-9. https:/ / doi.org/ 10.1038/nature04153

22. Endo A, Abbott S, Kucharski AJ, Funk S; Centre for the Mathematical Modelling of Infectious Diseases COVID-19 Working Group. Estimating the overdispersion in COVID-19 transmission using outbreak sizes outside China. Wellcome Open Res. 2020;5:67. https://doi.org/10.12688/ wellcomeopenres.15842.3

23. Althaus CL. Ebola superspreading. Lancet Infect Dis. 2015; 15:507-8. https://doi.org/10.1016/S1473-3099(15)70135-0

24. Böhning D, Rocchetti I, Maruotti A, Holling H. Estimating the undetected infections in the Covid-19 outbreak by harnessing capture-recapture methods. Int J Infect Dis. 2020;97:197-201. https://doi.org/10.1016/ j.ijid.2020.06.009

25. Alfò M, Böhning D, Rocchetti I. Upper bound estimators of the population size based on ordinal models for capturerecapture experiments. Biometrics. 2021;77:237-48. https://doi.org/10.1111/biom.13265

26. Jombart T, Jarvis CI, Mesfin S, Tabal N, Mossoko M, Mpia LM, et al. The cost of insecurity: from flare-up to control of a major Ebola virus disease hotspot during the outbreak in the Democratic Republic of the Congo, 2019. Euro Surveill. 2020;25:1900735. https:/ / doi.org/ 10.2807/1560-7917.ES.2020.25.2.1900735

27. Senga M, Koi A, Moses L, Wauquier N, Barboza P, Fernandez-Garcia MD, et al. Contact tracing performance during the Ebola virus disease outbreak in Kenema district, Sierra Leone. Philos Trans R Soc Lond B Biol Sci. 2017;372:372. https://doi.org/10.1098/rstb.2016.0300

28. Olu OO, Lamunu M, Nanyunja M, Dafae F, Samba T, Sempiira N, et al. Contact tracing during an outbreak of Ebola virus disease in the Western Area Districts of Sierra Leone: lessons for future Ebola outbreak response. Front Public Health. 2016;4:130. https:/ / doi.org/10.3389/ fpubh.2016.00130

29. Masumbuko Claude K, Underschultz J, Hawkes MT. Social resistance drives persistent transmission of Ebola virus 
disease in eastern Democratic Republic of Congo: a mixed-methods study. PLoS One. 2019;14:e0223104. https://doi.org/10.1371/journal.pone.0223104

30. Kiti MC, Kinyanjui TM, Koech DC, Munywoki PK, Medley GF, Nokes DJ. Quantifying age-related rates of social contact using diaries in a rural coastal population of Kenya. PLoS One. 2014;9:e104786. https://doi.org/10.1371/ journal.pone.0104786

31. Johnstone-Robertson SP, Mark D, Morrow C, Middelkoop K, Chiswell M, Aquino LDH, et al. Social mixing patterns within a South African township community: implications for respiratory disease transmission and control. Am J Epidemiol. 2011;174:1246-55. https://doi.org/10.1093/aje/kwr251

32. le Polain de Waroux O, Cohuet S, Ndazima D, Kucharski AJ, Juan-Giner A, Flasche S, et al. Characteristics of human encounters and social mixing patterns relevant to infectious diseases spread by close contact: a survey in
Southwest Uganda. BMC Infect Dis. 2018;18:172. https://doi.org/10.1186/s12879-018-3073-1

33. Kangbai JB. Social network analysis and modeling of cellphone-based syndromic surveillance data for Ebola in Sierra Leone. Asian Pac J Trop Med. 2016;9:851-5. https://doi.org/10.1016/j.apjtm.2016.07.005

34. Hagel C, Weidemann F, Gauch S, Edwards S, Tinnemann P. Analysing published global Ebola virus disease research using social network analysis. PLoS Negl Trop Dis. 2017;11:e0005747. https:/ / doi.org/10.1371/journal. pntd.0005747

Address for correspondence: Victor J. Del Rio Vilas, World Health Organization, South East Asia Regional Office, World Health House, Indraprastha Estate, Mahatma Gandhi Marg, New Delhi 110 002, India; email: delriov@who.int

\section{World AIDS Day, December 1}

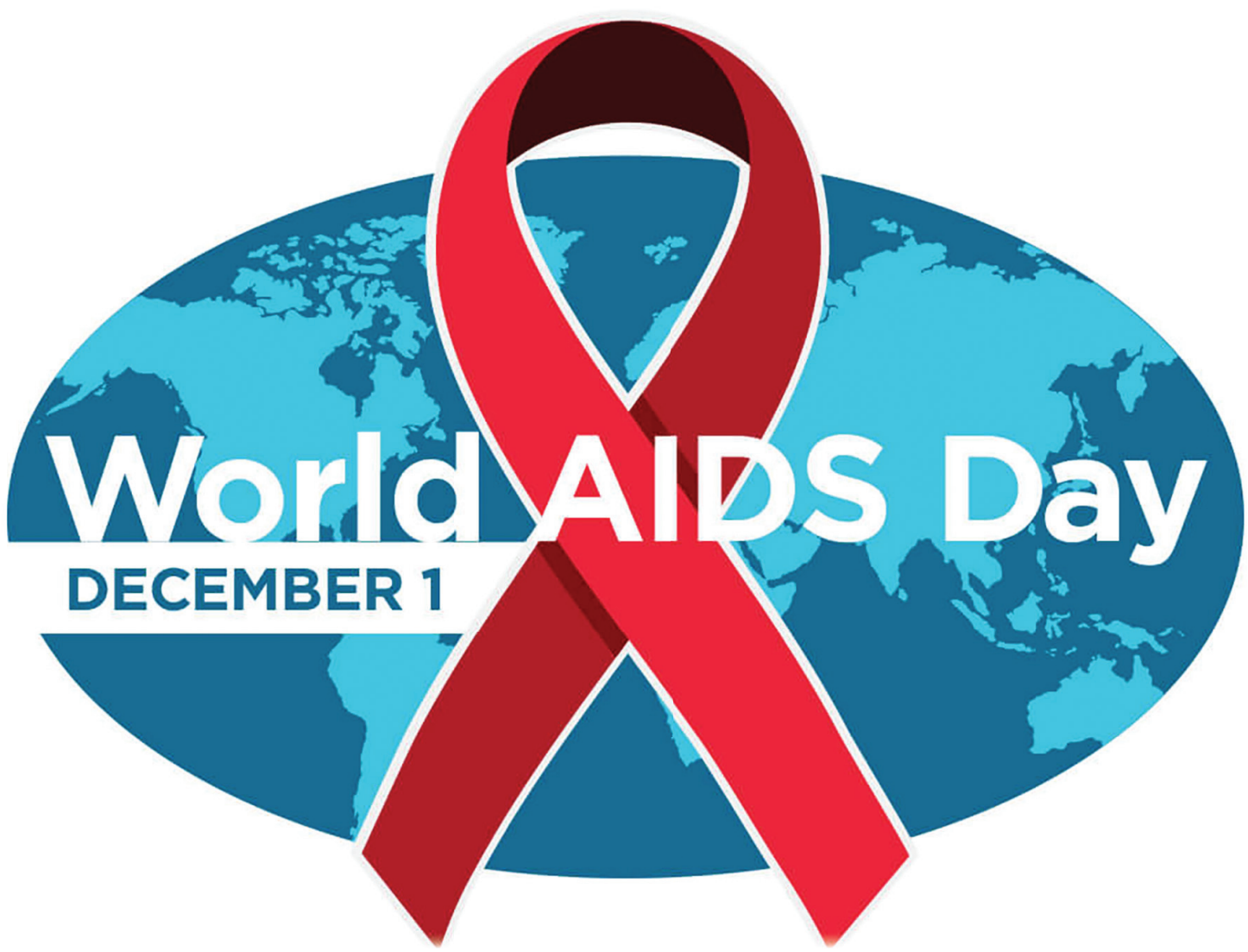

December 1 is World AIDS Day, an opportunity for people to work actively and collaboratively with partners around the world to raise awareness about HIV and help us move closer to the goal of an AIDS-free generation. The U.S. Government's theme for World AIDS Day 2021 is Ending the HIV Epidemic: Equitable Access, Everyone's Voice.

\section{http://wwwnc.cdc.gov/eid/page/world-aids EMERGING}

